\title{
$\angle S$ Research Square \\ Elective Bariatric and Metabolic Surgery during the COVID-19 Pandemic: Experience from Taiwan
}

\section{Darwin Salonga}

Body Science and Metabolic Disorders International Medical Center https://orcid.org/0000-0001-53535381

Chetan Parmar

Whittington Hospital

Ming-Che Hsin

Body Science and Metabolic Disorders International Medical Center

Chia-Chia Liu

Body Science and Metabolic Disorders International Medical Center

Yen-Chou Chen

Body Science and Metabolic Disorders International Medical Center

Chih-Kun Huang ( $\sim$ dr.ckhuang@hotmail.com )

Body Science and Metabolic Disorders International Medical Center

\section{Research Article}

Keywords: COVID-19, SAR-CoV2, Elective Bariatric and Metabolic Surgery

Posted Date: June 5th, 2020

DOI: https://doi.org/10.21203/rs.3.rs-32513/v1

License: (9) This work is licensed under a Creative Commons Attribution 4.0 International License.

Read Full License 


\section{Abstract}

Background: This study review the impact of an evolving pandemic on our elective operations, the timely and necessary precautions made by our government and institution to prevent and contain its spread.

Methods: We performed a retrospective review of all Elective Bariatric and Metabolic Surgery done at our center from $1^{\text {st }}$ January to $30^{\text {th }}$ April 2020 compared to the same time period last year. A summary of the pathway for patients presenting to the hospital and healthcare worker surveillance was done.

Results: Total of 99 patients underwent elective operations from $1^{\text {st }}$ January $-30^{\text {th }}$ April 2020 . There were 59 females and 40 males with an average BMI of $35.20 \mathrm{~kg} / \mathrm{m}^{2}$ and $40.68 \mathrm{~kg} / \mathrm{m}^{2}$ respectively. Compared from the same period last year with a total 117 patients, a decline of 18 elective operations (-15.38\%) was noted. All patients were properly screened for COVID-19. There was no reported case of post-operative fever of unknown etiology and/or upper respiratory infections developing COVID-19. There were no reported mortality. No surgeons or any healthcare worker in our center reported with COVID-19. We continue to do and offer elective operations, offer online and telephone consultation and follow up.

Conclusion: Elective operations do not need to be postponed if you have early mitigation measures of a pandemic spread, prompt implementation of protocols and strict adherence to these measures.

\section{Introduction}

The first four reported cases of the novel Coronavirus Disease (COVID-19) were seen on December 29, 2019 from local hospitals in Wuhan, Hubei Province, China [1]. Within 24 hours, Taiwan officials went on to check arriving planes from Wuhan to assess passengers with fever, respiratory symptoms, and stringent contact history before deplaning in active effort to mitigate the spread of the disease [2]. From these initial cases, the Severe Acute Respiratory Syndrome Coronavirus 2 (SARS-CoV-2) has grown to become a pandemic, has reached 200 countries worldwide and has more than 3.4 million confirmed cases, causing almost 240,000 deaths [3]. International Federation for the Surgery of Obesity and Metabolic Disorders (IFSO) have recommended postponement of all elective Bariatric and Metabolic Surgery (BMS) during the pandemic [4]. It was recommended to use only essential items for emergency and urgent cases to minimize the risk of Healthcare workers (HCW) transmission. As of April 8, 2020, nearly 22,073 cases of infected HCW from 52 countries had been reported by the World Health Organization (WHO) [5].

In this study, we review the impact of an evolving pandemic on our elective operations, the timely and necessary precautions made by our government and institution to prevent and contain its spread and finally, suggestions to adapt to these critical times.

\section{Preparations for the Pandemic}


Containing an outbreak at an early stage affords a greater chance of success than does a delayed response and clearly puts less strain on the healthcare system. In 2004, the year after the SARS outbreak, the Taiwan government established the National Health Command Center (NHCC). It includes the Central Epidemic Command Center (CECC), the Biological Pathogen Disaster Command Center, the CounterBioterrorism Command Center, and the Central Medical Emergency Operations Center [6]. It is part of a disaster management center that focuses on large-outbreak response and acts as the operational command point for direct communications among central, regional, and local authorities. Taiwan from its experience, has a head start of 16 years in terms of pandemic protocols, chain of command and systematic network of communications among government agencies and hospitals [6].

\section{Early key decisions, Detection and Mitigation}

On January 05,2020, CECC had started reporting passengers from Wuhan with fever and/or upper respiratory tract infections with issuance of flight Alert Level 2 and 3. On January 20, the CECC was fully activated and all its agencies had started analyzing data coming inside and outside of Taiwan. On $21^{\text {st }}$ January, the CECC confirmed its first case of COVID19, a 55-year-old female born in Southern Taiwan with a history of travel from Wuhan, making her the world's first non-Chinese national diagnosed with the disease [7]. On $23^{\text {rd }}$ January, Wuhan residents were banned and travellers from China were made to submit electronic declaration of health prior to entry. This was followed by bans of direct flights from provinces of Guangdong and Zhejiang and tour groups to Hongkong and Macau were suspended. By $6^{\text {th }}$ February, all Chinese nationals and International cruise ships were banned. Foreign nationals with travel to China, Hong Kong, or Macau in the past 14-days were likewise banned [2].

\section{Healthcare Policies}

On $31^{\text {st }}$ January, Taiwan government hastened production of surgical masks in anticipation of its increase in demand, to protect the HCW and most importantly to prevent the spread of the virus. They distributed and limited 3 surgical mask per person per week to all citizens. By February 02, they were able to produce 10 million mask/day. Commercial stores and pharmacies are not allowed to sell surgical masks [2]. Taiwan was one of the first nations to ban its HCW from non-essential travel overseas by February 24th. They have not only timely prevented the possible exposure of HCWs from other countries who might have early COVID-19 cases or asymptomatic carriers but likewise anticipated the increase in need of this essential human resources in time of pandemic. All HCWs in BMIMC are provided with official scrub suits that are solely maintained by the hospital itself. Upon entry in the hospital, they must change their attires. Upon exit from the operating room, they are to leave with a different color coded scrub suits. Upon end of rounds, clinic and/or ward work, scrub suits are left in the hospitals. These measures minimize possible viral load both going inside and outside the center from our HCWs attires. All $\mathrm{HCWs}$ are required to wear surgical masks both inside and outside the hospital (depending on Taiwan government daily advisory). The Infectious Control Committee of the China Medical University Hospital (CMU) gathered travel history of all HCWs for the past 14 days beginning February 24. All HCWs with any risk factors and/or having signs and symptoms of fever, difficulty of breathing and upper respiratory tract 
infection (URTI) are advised to go to the CMUH-Emergency Room (ER)-Isolation Tents. Mandatory COVID19 testing via Reverse transcription polymerase chain reaction (RT-PCR) were likewise done for HCWs with travel history to Alert Level 3 countries. If found positive results, they are managed accordingly and if found negative, they are advised home quarantine for 14 days, record their temperatures twice a day and watch out for signs and symptoms of URTI before returning to work.

\section{Unified Measures}

One Entrance: One Exit. CMUH building has directed the inflow and outflow of all patients in a single designated entrance and exit. This has narrowed the possibility of missing a possible COVID-19 suspected case if there were multiple entrance and exits. Thermal scanners were strategically placed, a confirmatory individual checking of body temperature via contactless thermometer. Patients with more than $36.8^{\circ} \mathrm{C}$ are not allowed to enter the building and directed to the CMUH ER as mentioned. All patients and their companion's hands are sprayed with 70\% alcohol. Taiwan has only 429 confirmed cases, 6 deaths ( $1.39 \%$ case fatality rate), 322 recoveries $(75.05 \%$ recovery rate) and has no documented "community transmission" of COVID-19 [8]. On April 30, 2020, Taiwan CECC has reported 6 consecutive days without a new confirmed case and a total of 19 days for April without local transmission [9]. Part of the success behind Taiwan's fight against COVID-19 is the positive outlook, dynamism, learning from the past experience and initiative of the people of Taiwan and compliance with the government's efforts to fight the disease according to Premier Su [10]. Using Big Data Analytics, Taiwan has successfully contained the spread of the SARS-COV-2 by mobile position data (Contact Tracing). In conjunction with GPS, credit card transaction, and health record they are able to locate a COVID-suspect person and they are able to identify those who had contact with him/her within 500 meters leading to an effective contact tracing. Taiwan has likewise intensified its search for possible unidentified COVID-19 cases. By reviewing all medical health record of patients in National Health Insurance database, they can establish who were hospitalized due to pneumonia. For those hospitalized but had not been tested for SARS-CoV-2, the local health care providers were informed and screening for SARS-CoV-2 was suggested [11].

\section{Method}

We performed a retrospective review of all BMS done at the Body science at Metabolic disorders International Medical Center (BMIMC) at CMUH from $1^{\text {st }}$ January to $30^{\text {th }}$ April 2020 encompassing 4 months, analyzed and compared with the cases from the same period last year. Figure 1.0 summarizes the pathway for patients presenting to our hospital. At a single designated entrance of our building, all patients are screened for history of travel, fever and/or any upper respiratory symptoms within 14 days. Patients with above findings are not allowed to enter BMIMC and directed to an Isolation Area outside the CMUH ER - Isolation Tents for thorough assessment. COVID-19 suspected cases are tested with RT-PCR via nasopharyngeal swab sample and portable chest X-ray (CXR) was likewise done with initial reporting done by a radiologist. Results are released via private text message with a turn-around time of 6 to 8 hours. They are managed accordingly based on these screening tools. Asymptomatic BMIMC patients for initial appointment, follow-up or elective surgery but with travel history to countries with known COVID-19 
community transmission are advised to undergo 14-days home quarantine prior to scheduling. Preoperative weights, body mass index (BMI) and co-morbidities of patients were obtained. All patients undergo pre-operative endoscopic gastroduodenoscopy (EGD), whole abdominal ultrasonography, electrocardiogram and routine CXR. Chest CT Scan is only performed if suspected findings on CXR or COVID-19 risk factors. Patients are seen by a multi-disciplinary team of dietician, psychologist, obesity medicine specialist and anaesthesiologist. All cases were done laparoscopically and staff wearing standard Personal Protective Equipment (PPE). The operating theatre has High-efficiency particular air (HEPA) filter and standard positive pressure operating room.

\section{Results And Discussions}

\section{Demographics}

Total of 99 patients underwent elective BMS from $1^{\text {st }}$ January $-30^{\text {th }}$ April 2020. All patients underwent complete pre-operative evaluation as stated. There were 59 females and 40 males with an average BMI of $35.20 \mathrm{~kg} / \mathrm{m}^{2}$ and $40.68 \mathrm{~kg} / \mathrm{m}^{2}$ respectively. Comparing with our data from the same period last year, we had a total 117 patients undergoing elective BMS and hence noticed a decline of 18 patients or $15.38 \%$ in total. We had 83 females and 34 males with average BMI of $35.49 \mathrm{~kg} / \mathrm{m}^{2}$ and with $39.99 \mathrm{~kg} / \mathrm{m}^{2}$ respectively. Most common co-morbidities are: ( $n=99)$ : Hypertension (50), Hyperlipidaemia (50), Hyperuricemia (34), Fatty Liver (34) and Diabetes Mellitus (29).

\section{Preoperative Screening}

All patients underwent mandatory clearance for COVID-19 with the following criteria within the previous 14 days: No history of travel to countries with known COVID-19 confirmed cases, no fever, no URTI signs and symptoms. Mandatory Infectious Disease clearance was given for patients with prior history and risk factors. Negative RT-PCR results at day 7 and day 14 of disease were needed to clear suspected and/or confirmed COVID-19 cases. Hence, all patients were fully cleared prior to scheduled surgery.

\section{Types of Elective Operations performed}

Comparison of elective BMS done in 2019 vs 2020 showed Laparoscopic Sleeve Gastrectomy (LSG) 68 vs 51; Laparoscopic Proximal Jejunal Bypass with Sleeve Gastrectomy (LPJBSG) 32 vs 34; Laparoscopic Duodenojejunal Bypass with Sleeve Gastrectomy (LDJSG) 2 vs 3; Laparoscopic Roux en Y Gastric Bypass (LRYGB) 8 vs 4 and Revisional Surgeries 7 vs 7. Based on the type of BMS, LSG has the most number of declined case (17 or $25 \%$ ).

\section{Trend of Elective Operations performed}

Figure 2.0 shows the summary based on the monthly Census in 2019 vs 2020 . During the announcement of pandemic on March by WHO, interestingly the total census for the month even increased by $37.5 \%$, as most of our patients noted the safety and felt undue need to postpone elective BMS. The month of April 
this year had the most decline of elective BMS (-52.5\%) mainly due to the travel restrictions outside Taiwan due to the global outbreak. From 15 international patients in 2019, we only had 4 this year. Another likely explanation is the decline in global and local economy and fear of exposure to the virus. With a total of 117 cases in 2019 vs 99 in 2020 from the same time, there is just $15.4 \%$ decrease in total census of elective BMS. Given the IFSO recommendation to stop all elective BMS, timely and wellcoordinated prevention of the COVID-19 and thorough screening are the most important factors containing it's spread, maintain patient safety and prevent unnecessary economic losses. A robust protocol needs to be followed by the hospital (Figure 1).

\section{Complications of Elective BMS during the Pandemic}

There was no reported case of post-operative patients developing fever of unknown etiology, upper respiratory infections, hence none of our post-operative patients needed COVID-19 testing. There was one case of staple leak from an LSG patient with Cushing's Syndrome which was successfully managed by re-operation. There was no reported mortality. There was no difference in the length of hospital stay for both groups. Follow up was done by clinic visit, telephone or online (LINE, Facebook messengers).

\section{Healthcare Worker Infection}

It is important to note, that we routinely use standard PPE in our elective BMS and not full PPE, but till now no surgeon or HCWs have been reported to be infected with COVID-19 in our center. Figure 3.0 shows the pathway for our HCWs protection and surveillance.

\section{Conclusion}

Our experience highlights that with stringent and well-planned healthcare policies, good governance and cooperation of all sectors, Taiwan has contained the adverse effects on the healthcare. Elective Bariatric and Metabolic Surgeries do not need to be postponed if you have early mitigation measures of a pandemic spread, protocols for network and analysis of data, and proper execution of these measures. To our knowledge we are the only unit in the world to perform elective BMS during the COVID19 pandemic. The safety of both the patient and healthcare team have been of prime importance during this time of pandemic and Taiwan and our bariatric center has been exemplary in this field and can be possibly used as a model system for future pandemic outbreaks.

\section{Declarations}

Ethical Approval: This article does not contain any studies with human participants or animals performed by any of the authors.

Conflict of Interest: The authors declare that they have no conflict of interest.

Informed consent: For this type of study formal consent is not required. 
Ethical Approval and Informed Consent: All procedures performed in the study were in accordance of the institution's ethics committee and with the 1964 Helsinki declaration and its later amendments. The ethics committee waived the need for a formal informed consent due to the retrospective nature of the study.

\section{References}

1. Li Q, Med. M, Guan X, Wu P, Wang X, Zhou L, et.al., Early Transmission Dynamics in Wuhan, China, of Novel Coronavirus-Infected Pneumonia. The New England Journal of Medicine 2020; 382:11991207

2. Wang C, Ng C and Brook R. Response to COVID-19 in Taiwan: Big Data Analytics, New Technology, and Proactive Testing. JAMA. Published online March 03, 2020. [cited 2020 Apr 15] Available from https://jamanetwork.com/journals/jama/fullarticle/2762689

3. WHO Director-General's remarks at the media briefing on 2019-nCoV on 11 February 2020. [cited 2020 Apr 16] Available from https://www.who.int/dg/speeches/detail/who-director-general-sremarks-at-the-media-briefing-on-2019-ncov-on-11-february-2020.

4. Yang, W., Wang, C., Shikora, S. et al. Recommendations for Metabolic and Bariatric Surgery During the COVID-19 Pandemic from IFSO. OBES SURG 30, 2071-2073 (2020). https://doi.org/10.1007/s11695-020-04578-1

5. Coronavirus disease 2019 (COVID-19) Situation Report - 82. [cited by 2020 Apr 15] Available from https://www.who.int/docs/default-source/coronaviruse/situation-reports/20200411-sitrep-82-covid19.pdf?sfvrsn=74a5d15_2.

6. Chen K, Twu S, Chang H, Yi-Chun W, Chen C, Lin T, et al., SARS in Taiwan: an overview and lessons learned, Int. J. Infect. Dis. 9 (2) (2005) 77-85.

7. Cheng S, Chang Y, Chiang Y, et.al., First case of Coronavirus Disease 2019 (COVID-19) pneumonia in Taiwan, Journal of the Formosan Medical Association, Volume 119, Issue 3, 2020, 747-751

8. Taiwan Center for Disease Control Coronavirus Situation. [cited 2020 Apr 30] Available from https://www.cdc.gov.tw/En.

9. Taiwan records 6 consecutive days without new coronavirus cases. [cited 2020 May 01] Available from https://www.taiwannews.com.tw/en/news/3926386.

10. Premier promotes new focus on personal responsibility to contain COVID-19. [cited 2020 May 01] Available from https://english.ey.gov.tw/Page/61BF20C3E89B856/fa513756-e6e5-4336-a1b66801801abfaf.

11. Chen CM, Jyan HW, Chien SC, Jen HH, Hsu CY, Lee PC, et. Al., Containing COVID-19 Among 627,386 Persons in Contact With the Diamond Princess Cruise Ship Passengers Who Disembarked in Taiwan: Big Data Analytics. J Med Internet Res 2020;22(5):e19540 DOI: 2196/19540 PMID: 32353827

\section{Figures}




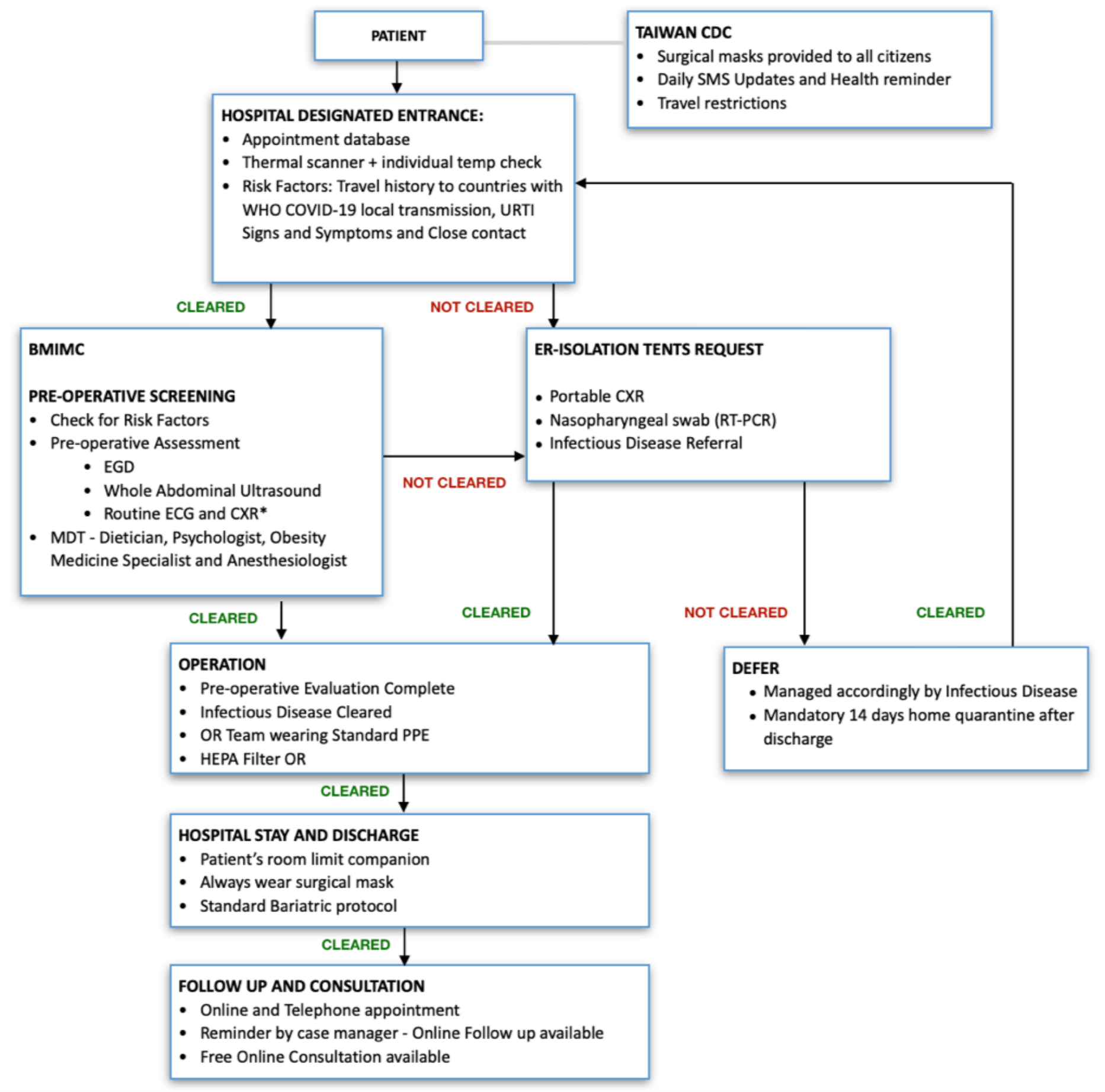

\section{Figure 1}

Patient Pathway presenting to the hospital for Elective Bariatric and Metabolic Surgery. ${ }^{*} \mathrm{CXR}$ - Additional Chest CT Scan is requested. Abbreviations: SMS, short messaging system; temp, temperature; WHO, World Health Organization; COVID-19, novel coronavirus disease; URTI, upper respiratory tract infections; EGD, esophagogastroduodenoscopy; ECG, echocardiogram; CXR, chest X-ray; RT-PCR, Reverse transcription polymerase chain reaction; OR, Operating Room; PPE, Personal Protective Equipment; HEPA. High-efficiency particulate air. 


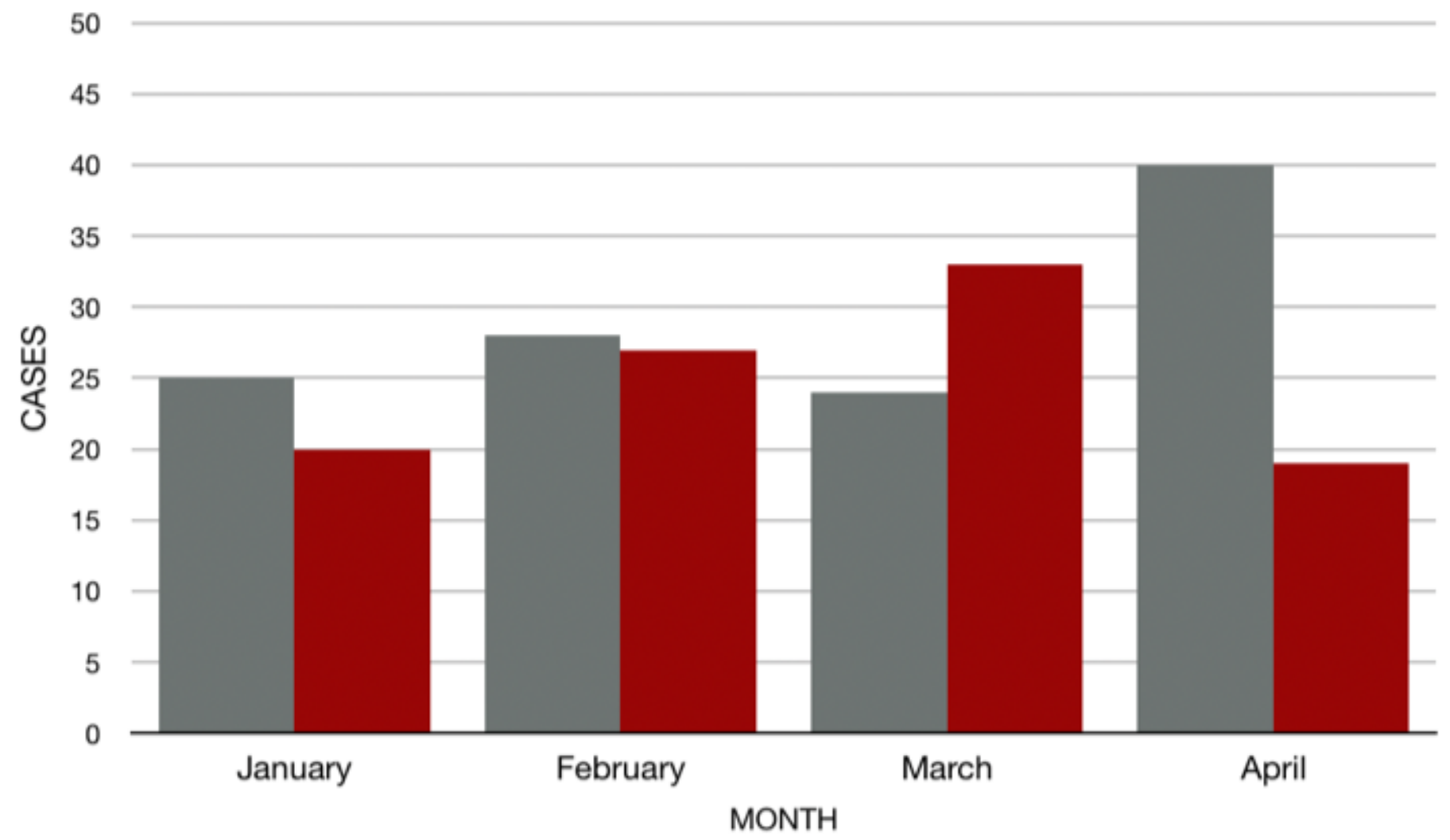

Figure 2

Summary of Elective Bariatric and Metabolic cases per month, 2019 vs 2020. 


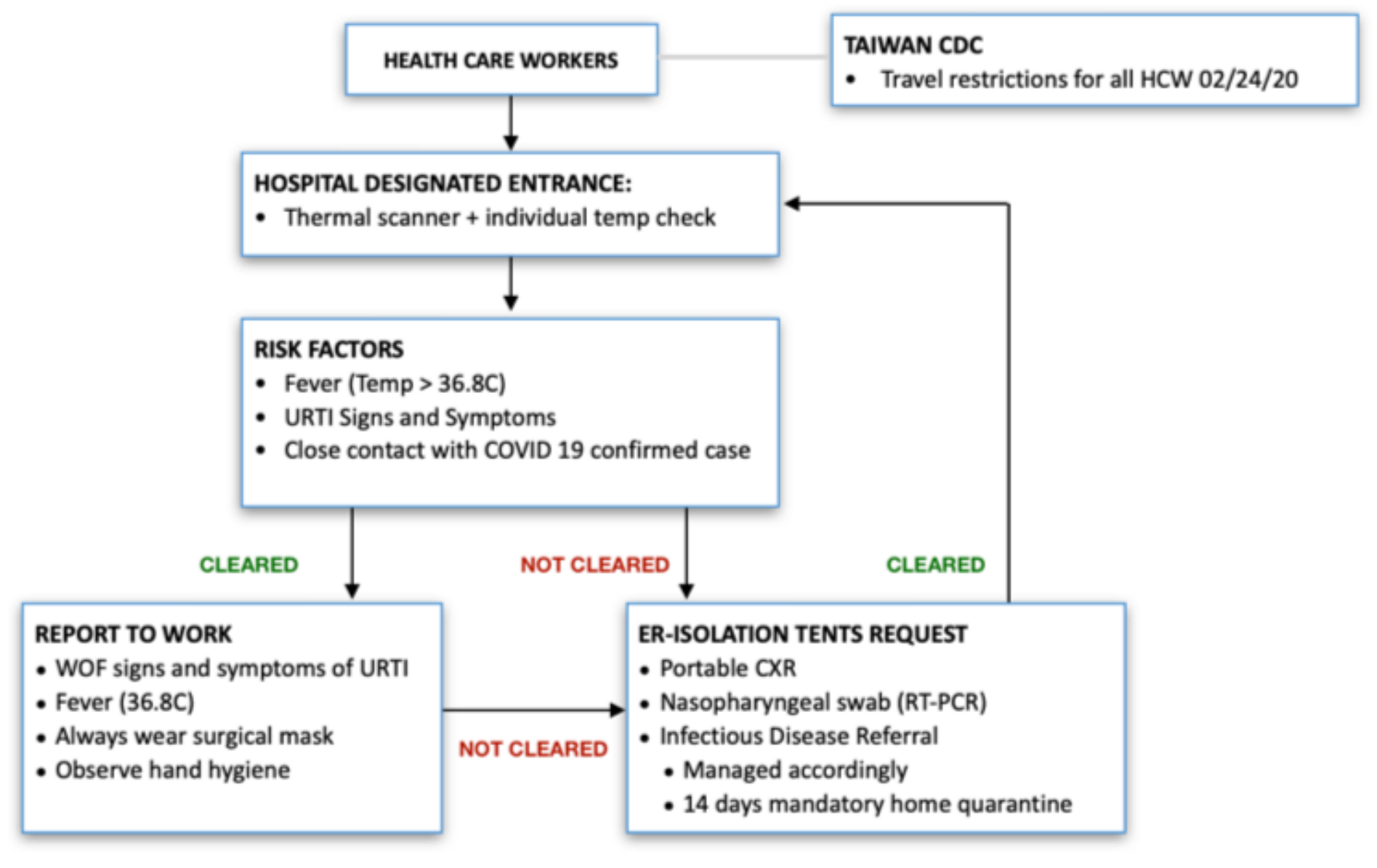

\section{Figure 3}

Healthcare workers pathway for protection and surveillance. Abbreviations: HCW, Healthcare workers; Temp, temperature; URTI, upper respiratory tract infections; COVID-19, novel coronavirus disease; RT-PCR, Reverse transcription polymerase chain reaction. 